\title{
EMPATÍA Y PROSOCIALIDAD: PROYECTOS DE APRENDIZAJE-SERVICIO EN PSICOLOGÍA SOCIAL
}

\author{
Yolanda Ruiz Ordóñez \\ Universidad Católica de Valencia \\ S. Vicente Mártir (Valencia) \\ yolanda.ruiz@ucv.es
}

Recepción Artículo: 18 enero 2020 Admisión Evaluación: 4 marzo 2020 Informe Evaluador 1: 13 marzo2020

Informe Evaluador 2: 18 marzo2020

Aprobación Publicación: 20 abril 2020

\section{RESUMEN}

Los proyectos de Aprendizaje-Servicio son una oportunidad para trabajar tanto la empatía como la prosocialidad de los universitarios. Trasladar contenidos teóricos al contexto real de manera práctica contribuye a la adquisición de competencias transversales de las asignaturas.

El objetivo de este trabajo es presentar experiencias realizadas con esta metodología por un grupo de alumnos de la asignatura de psicología social. Estos Ilevaron a cabo varios proyectos con diferentes entidades, aunque todas ellas se centraron en dar respuesta a necesidades de niños y adolescentes en riesgo de exclusión social, procedentes de barrios problemáticos y de familias desestructuradas, sin apenas recursos para establecer relaciones interpersonales saludables.

En dichos contextos, los alumnos universitarios detectaron problemas de convivencia, falta de cohesión social, escasa inteligencia emocional, presencia de conductas disruptivas, desarrollo psicoafectivo escaso y frustración ante cuestiones académicas que afectaban a cuestiones tanto atencionales como conductuales. El proyecto de intervención de los alumnos para abordar esta problemática se llevó a cabo desde un trabajo en red con otras ONGs y asociaciones. También, se centró básicamente en reforzar las buenas prácticas que ya se estaban realizando en los centros educativos a través del refuerzo escolar, y se les enseñó a trabajar en equipo a través de juegos y actividades deportivas.

Los resultados de este servicio contribuyeron a mejorar en los niños y adolescentes su identidad social al trabajar el autoconcepto y la autoestima, fomentaron la autonomía y fortalecieron aptitudes para ayudarles a integrarse en la sociedad sin obviar la educación en valores como solidaridad, respeto y responsabilidad. Además, aprendieron herramientas como la negociación y el diálogo para solucionar sus conflictos en los distintos ámbitos de su vida. Y los alumnos valoraron positivamente la metodología, desarrollando así la 


\section{EMPATÍA Y PROSOCIALIDAD: PROYECTOS DE APRENDIZAJE-SERVICIO EN PSICOLOGÍA SOCIAL}

prosocialidad y la empatía, fruto de la experiencia de aprendizaje derivada de ésta tanto a nivel personal como académico.

Palabras clave: adolescencia; infancia; exclusión; prosocialidad; empatía

\section{ABSTRACT}

Empathy and prosociality: service-learning projects in social psychology. Service-learning projects are an opportunity to work on both the empathy and the prosociality of university students. Transferring theoretical content to the real context in a practical way contributes to the acquisition of transversal skills of the subjects.

The aim of this paper is to present experiences made with this methodology by a group of students of the subject of social psychology. They carried out several projects with different entities, although all of them were focused on responding to the needs of children and adolescents at risk of social exclusion, coming from problematic neighbourhoods and unstructured families, with hardly any resources to establish healthy interpersonal relationships.

In these contexts, the university students detected problems of coexistence, lack of social cohesion, low emotional intelligence, presence of disruptive behaviors, scarce psycho-affective development and frustration with academic issues that affected both attention and behavior. The students' intervention project to address this problem was carried out through networking with other NGOs and associations. It also focused basically on reinforcing the good practices that were already being carried out in the schools through school reinforcement, and they were taught to work as a team through games and sports activities.

The results of this service contributed to improving children and adolescents' social identity by working on their self-concept and self-esteem, promoting autonomy and strengthening skills to help them integrate into society without neglecting education in values such as solidarity, respect and responsibility. In addition, they learned tools such as negotiation and dialogue to solve their conflicts in the different areas of their lives. And the students valued the methodology positively, thus developing prosociality and empathy, the result of the learning experience derived from it both on a personal and academic level.

Keywords: adolescence; childhood; exclusion; prosociality; empathy

\section{INTRODUCCIÓN}

En la actualidad, la comisión sectorial de sostenibilidad del Consejo de Rectores de Universidades Españolas de 2015 recomienda la activación de la metodología Aprendizaje-Servicio en el marco de la Universidad Española como clave para desarrollar la responsabilidad social para el aprendizaje de competencias que promuevan la participación social y la ciudadanía activa. Aprendizaje-Servicio debe ser planificado desde la Institución Educativa, contando con la participación de los docentes y estudiantes, y no únicamente con la demanda de la comunidad. Este tipo de metodología fomenta la calidad académica y promueve el compromiso social y actitudes prosociales. (Sandrea, 2008, p.83).

Estas actitudes se pueden trabajar desde una metodología experiencial y vivencial que refuercen aspectos como ayuda y servicio, solidaridad, asertividad, escucha activa, empatía, autoestima y creatividad. (Sandrea, y Reyes, 2010, p.382).

Roche (1995) plantea que los estudiantes pueden tener conductas prosociales preestablecidas, bien por el contacto directo con diferentes entidades sociales o bien sea por imitación. Pero también hay que destacar que incrementar la participación social implica incrementar la actitud prosocial porque, como afirma Coleman (1988), la participación de las personas en actividades de bien común contribuye a la formación de capital social (Sandrea y Reyes, 2010, p.389-390).

La prosocialidad se define como "aquellas acciones que tienden a beneficiar a otras personas sin la expectativa de beneficio personal externo". (Eberly, 2002, p.2). Comprenden un conjunto de conductas que permiten manejar valores adquiridos mediante Aprendizaje-Servicio. Se pensaba que la prosocialidad respondía a un com- 
portamiento a favor de los destinatarios, pero aun aceptando esta afirmación, se considera que también posee beneficios importantes sobre uno mismo, teniendo de este modo una influencia positiva en la personalidad, autoestima y empatía de quien pone en práctica este comportamiento. Esta metodología es un buen método para educar en prosocialidad, estableciéndose asimismo un proceso que retroalimenta la participación y la implicación en este tipo de proyectos.

Las conductas que explican la prosocialidad son ayuda y servicio físico, es decir, cumplir un objetivo respondiendo a una necesidad del beneficiario; dar y compartir; ayuda y consuelo verbal; valoración positiva del otro, esto es, reconocimiento de la otra persona, escucha profunda, solidaridad y presencia personal positiva y empatía. (Puig, Batllé y Palos, 2006, p. 48). Esta última se considera una de las conductas verbales que se vinculan con este tipo de comportamientos y que se relaciona con la expresión tanto cognitiva como emocional. (Eberly, 2002, p.2-3).

Barrio y otros (2004) han concluido que los factores estructurales de la personalidad, de amistad y de conciencia son los que tienen mayor peso en las conductas prosociales y en la capacidad del sujeto de empatizar y cooperar ante las necesidades de los otros, junto con la aptitud de auto-regulación y de autoorganización de la conducta que también se relacionan con la prosocialidad. Carlo y otros (1999) confirman que el desarrollo prosocial es un asunto complejo que depende de una multiplicidad de factores interrelacionados como la cultura, el contexto familiar, el ámbito escolar, factores cognitivos, afectivos y de sociabilidad, entre otros. (Sandrea y Reyes, 2010, p.390).

El servicio activa el dinamismo de la conducta prosocial porque son los propios alumnos los que lideran los cambios del entorno social. Además, "desarrollar la gratuidad y el altruismo es imprescindible para establecer vínculos sociales y sentar las bases de una ciudadanía democrática comprometida con el futuro de la humanidad" (Martín, 2018, p. 47).

Pero también "Aprendizaje-Servicio es una educación de las emociones y de la empatía" (Ardila y Correa, 2018, p.271). La empatía es la capacidad que se tiene para ponerse en el lugar del otro y ayuda a modular la conducta prosocial frente a conductas agresivas (Sandrea, y Reyes, 2010, p.390).

Los jóvenes tienen capacidad de adquirir ciertas habilidades no solo para recibir, sino para dar lo que han recibido de la sociedad. Hacer que aporten algo valioso a la comunidad forma parte de todo proceso formativo. Por eso, metodologías como Aprendizaje-Servicio son útiles, porque ponen en valor la importancia de solicitarles ayuda. Cuando son conscientes de esto, se empoderan y refuerzan su identidad. Por tanto tiene efectos positivos, ya que la conducta de donación implica un agradecimiento por parte de los receptores de su acción, al mismo tiempo que les hace sentir partícipes activos de la comunidad incrementando, de este modo, su autoestima. (Martin, 2018, p. 41).

La nueva situación de la universidad nos plantea nuevos retos y un nuevo modelo pedagógico que oriente la investigación y la docencia hacia el bien común y supere posturas reduccionistas. (Gil y Reyero, 2015, p. 5455). Aplicar esta metodología supone un cambio en la concepción de la propia universidad y afecta directamente a los profesores porque "solo enseñará realmente a aprender el que aprende de su enseñar, es decir, el que somete a reflexión y crítica permanente su ejercicio de enseñanza para convertirlo también en ejercicio de aprendizaje" (Pérez, 2007, p.159).

\section{OBJETIVOS DE LA INVESTIGACIÓN}

El objetivo principal es promover la empatía y las conductas prosociales en alumnos de la asignatura de psicología social de segundo curso en el Grado de psicología mediante la participación en proyectos de Aprendizaje-Servicio. Para ello se plantean como objetivos específicos: establecer una relación directa de los contenidos la asignatura de psicología social con el contexto real, aprender a diagnosticar la realidad desarrollando la capacidad de observación, adquirir competencias de la asignatura en contexto real y, generar una mayor sensibilidad y actitud positiva hacia la disciplina, visibilizando su carácter práctico desde una metodología experiencial. 


\section{EMPATÍA Y PROSOCIALIDAD: PROYECTOS DE APRENDIZAJE-SERVICIO EN PSICOLOGÍA SOCIAL}

\section{METODOLOGÍA}

La propuesta metodológica se centra en activar Aprendizaje-Servicio en tres grupos de alumnos que actúen sobre niños y adolescentes en situaciones de exclusión y vulnerabilidad. De este modo, las asociaciones destinatarias situadas en Valencia son Amaltea, Entre Amics y Colegio Santiago Apóstol.

Los datos recientes nos indican que 1 de cada 4 menores de 16 años están o estarán en riesgo de exclusión social, de manera que no podrán satisfacer sus necesidades como es el acceso a una educación, a una vivienda, a tener un trabajo y a participar en la sociedad. Concretamente, si atendemos a las cifras proporcionadas según Ios últimos datos publicados por el Instituto de Estadísticas (IVE) y citados por Save the Children, cerca del 33\% de menores de 16 años se encuentran en la comunidad valenciana en riesgo de exclusión social, siendo el colectivo más vulnerable.

La asociación Amaltea trabaja con adolescentes que están en exclusión al petenecer a familias con una situación económica y laboral precaria e inestable. Muchos tienen experiencias negativas como maltrato o consumo de sustancias, así como trastornos tanto emocionales como conductuales. Predomina la inestabilidad emocional y la conducta agresiva.

Ante esta situación, un grupo de alumnos universitarios se ha centrado en reducir las conductas violentas e irascibles mediante actividades que promuevan el respeto a los demás, utilizando el refuerzo como técnica de modificación de conducta. De este Aprendizaje-Servicio se espera como resultados que los adolescentes de Amaltea hagan uso de la negociación y el diálogo como herramienta para responder, sin violencia, a situaciones estresantes y a solucionar conflictos, pero no solo en el ámbito académico, sino también en su vida diaria.

La asociación "Entre Amics" pertenece a S. Juan Bosco. Está situada en una zona de exclusión y caracterizada por la presencia significativa de población analfabeta y de familias con problemas de desestructuración familiar grave, al que se añade el consumo de alcohol y drogas. Se trata pues de una zona de atención educativa preferente. Los jóvenes que viven allí están en situación de riesgo social, ocupando su tiempo de ocio con el consumo de sustancias y con el establecimiento de relaciones interpersonales negativas.

En este contexto se sitúa Entre Amics, un centro destinado a menores en situación de riesgo y exclusión social de 6 a 16 años. Acoge principalmente, y con carácter de prioridad, a menores que proceden de una resolución administrativa de los servicios territoriales del órgano especialista, de los centros escolares o institutos del barrio, de Servicios Sociales de Quatre Carreres, del servicio psicopedagógico escolar (SPE) y de Salud Mental infantil de la zona. También, la propia familia puede solicitarlo, pero en cualquier caso se valora si realmente existe una situación derivada de la ausencia o escasez de recursos económicos, sociales, culturales y sanitarios que incidan negativamente en el desarrollo integral de la persona.

Las áreas que se analizan para el acceso al centro son fundamentalmente las siguientes:

1. Desestructuración familiar, analizando si hay padres enfermos, divorciados, con problemas de adicciones y situaciones de orfandad 0 abandono.

2. Situación socio-económica, conociendo si existen realmente recursos básicos, si están en una zona de carencia social o si los padres están en paro laboral.

3. Fracaso educativo y escolar, ámbito que considera los problemas de disciplina, de desmotivación académica, de bajo rendimiento, de absentismo escolar e incluso de riesgo social a nivel económico, cultural, de violencia familiar e incluso de consumo de alcohol.

4. Desarrollo personal, teniendo presente cuestiones relativas a la introversión, dificultades de aprendizaje, deficiencias cognitivas y retrasos en el desarrollo.

5. Integración étnica-interculturalidad, valorando especialmente si proceden de algún grupo 0 etnia que pueda ser susceptible de discriminación o rechazo.

6. Deficiencias físicas, psíquicas y/o sensoriales, considerando cualquier tipo de necesidad educativa especial 0 de diversidad funcional que requiera ser atendida.

En este centro se pretende que el entorno educativo favorezca hábitos y conocimientos que permitan la integración. Se centra en una intervención socioeducativa, en fortalecer las aptitudes de las personas, en intervenir 
con las familias para que los menores puedan mejorar su rendimiento social y académico, y en colaborar con distintos centros de forma armonizada.

La problemática más acuciante que se detecta en los menores es la frustración ante los estudios, al mismo tiempo que incapacidad para trabajar en grupo. Además, su entorno familiar también afecta a su desarrollo psicoafectivo, dado que son muchos los que manifiestan problemas emocionales de apoyo.

Teniendo presente esta situación, el objetivo del Aprendizaje-Servicio de los alumnos de psicología se centró en la importancia de enseñarles a resolver conflictos a través de dos tipos de actividades: lúdicas y académicas con el fin de que pudieran afrontar los retos académicos. Sin embargo, se encontraron problemas de habilidades sociales y de conducta que, en ocasiones, afectaban al desarrollo de las actividades.

El tercer centro era el colegio Santiago Apóstol, Centro de Atención Educativa Singular, cuyos niveles educativos oscilan desde Educación Infantil a Formación Profesional Básica. Está ubicado en una zona periférica de Valencia capital, siendo el 95\% del alumnado del centro de etnia gitana.

La población de etnia gitana suele migrar de una región a otra, situación que incide en que cambien de un centro académico, aspecto que afecta a la adaptación al aula. Tienden a relacionarse entre ellos mismos, de manera que los niveles de socialización e integración deben ser trabajados. Además, son muchos los familiares que coinciden en la misma clase, hecho que afecta más al aislamiento social de estos alumnos. Las familias se desentienden de la educación de los hijos, justificándose así el absentismo.

Son pocos los alumnos de etnia gitana los que finalizan la Educación Secundaria Obligatoria y menos los que llegan a la Universidad. El centro arroja los siguientes datos: el 64\% del alumnado gitano de entre 16 y 24 años no concluye los estudios obligatorios, siendo la mayor tasa de abandono a los 16 años y en segundo de la Educación Secundaria Obligatoria. Respecto a las esperanzas de vida escolar, se espera que un chico de 12 años estudie hasta los 20,7 años y una chica hasta los 21,3 años. Cuando se trata de jóvenes de etnia gitana, la esperanza de vida escolar baja a 17,8 años para los chicos y hasta los 15,5 años para las chicas.

El centro escolar pretende crear un espacio de aprendizaje en el colegio para que los niños de etnia gitana puedan tener en un futuro una salida laboral que trasciendan los trabajos de chatarrería de los padres. Además, cuenta con una escuela "matinera" en la que se ofrecen a los usuarios la posibilidad de hacer uso de las duchas, servicio de desayuno y tratamiento antiparasitario.

La problemática del centro busca como respuesta aumentar la cohesión social en los barrios y poblaciones, fomentar la convivencia, trabajar la falta de motivación académica, incrementar la autoestima y autoconcepto para capacitarles para el mundo laboral. El tema del fracaso escolar es evidente, pues los padres tampoco invierten en recursos escolares necesarios al no disponer de recursos económicos. Hay que añadir que los alumnos carecen de un sitio fijo de estudio, de manera que no pueden realizar las tareas o deberes. De ahí que el interés del centro es reforzar las buenas prácticas y posibilitar el aprendizaje de los niños.

El objetivo que los alumnos universitarios plantearon en el proyecto se basaba en reforzar la motivación académica mediante el apoyo a tareas escolares, despertando el interés por la lectura, ayudando a la planificación y acción de actividades deportivas en primaria y, promoviendo un estilo de vida saludable. También, gestionaron y organizaron materiales en el centro al colaborar activamente con los profesores en la enseñanza.

La intervención se realizó mediante técnicas de modificación de conducta y mediante la aplicación de refuerzos y resolución de conflictos en el aula. Los alumnos utilizaban el sistema de economía de fichas proporcionada por los educadores para cooperar en la modificación de ciertas conductas negativas de lectura, así como en el uso de las tecnologías de información y comunicación.

Los estudiantes de psicología se ocuparon igualmente de trabajar las emociones. Les motivaban a reflexionar escribiendo situaciones en las que habían experimentado estas emociones para, posteriormente, exponerlo grupalmente. Trabajaron además valores como el respeto, la igualdad, la equidad, la tolerancia, el compañerismo, el trabajo en equipo y la honestidad mediante juegos y carteles que elaboraban como eslóganes publicitarios. Las habilidades estratégicas y lingüísticas se desarrollaron con la exposición de poesía y lectura en voz alta, para de este modo adquirir capacidades (teatro) y evitar/quitar miedos. Paralelamente se desarrollaron juegos de 


\section{EMPATÍA Y PROSOCIALIDAD: PROYECTOS DE APRENDIZAJE-SERVICIO EN PSICOLOGÍA SOCIAL}

simulación, talleres de aprendizaje y espacios de diálogo. Se pretendía mejorar relaciones sociales, promover la motivación académica mediante recompensas extrínsecas como intrínsecas, incrementando la confianza en ellos mismos.

\section{MUESTRA Y PARTICIPANTES}

Los participantes fueron 17 alumnos de segundo curso del Grado de la asignatura de Psicología Social de la Universidad Católica de Valencia S. Vicente Mártir. Realizaron su Aprendizaje-Servicio en las tres organizaciones anteriormente mencionadas, dirigiendo sus intervenciones a 60 alumnos comprendidos en edades de 6 a 16 años, intervalo de edad que dependía del centro en el que estaban.

\section{PROCEDIMIENTO}

La metodología Aprendizaje-Servicio se ha desarrollado con una etapa previa de motivación a los alumnos para que consideren la importancia de vincular la asignatura con la sociedad. Los alumnos configuran grupos de 3 a 6 personas y seleccionan los ámbitos en los que creen que podrían realizar el proyecto de AprendizajeServicio. En este caso, los diferentes grupos de alumnos han seleccionado niños y adolescentes en exclusión. Realizan una búsqueda de asociaciones o entidades que aborden dichos ámbitos con el fin de elegir aquella entidad que consideren podía ser más adecuada para realizar su Aprendizaje-Servicio. Después de observar y conocer la entidad a través de los profesionales, realizan una detección de necesidades con la finalidad de seleccionar aquella a la que pueden responder, siempre atendiendo a las competencias y resultados de aprendizaje de la asignatura. Posteriormente, configuran el proyecto fundamentando la relevancia del contexto sobre el que van a realizar su Aprendizaje-Servicio. Establecen los objetivos del proyecto para los que proponen una serie de acciones 0 actividades que podrán poner en práctica.

Por último, realizan la evaluación de su servicio por parte de todos los actores sociales implicados. Los espacios de reflexión establecidos de manera procesual y sistemática son esenciales para valorar la calidad del servicio tanto a nivel personal como grupal.

\section{RESULTADOS}

Los resultados que se lograron con esta metodología para los alumnos universitarios fue fomentar el aprendizaje y trazar el horizonte para el mundo laboral, acercándose tanto a la psicología social como a la psicología de la educación. Descubrieron nuevos conceptos, pero también nuevos modos de trabajar en los que el deporte y los juegos favorecían el aprendizaje y el trabajo en equipo. Paralelamente, el hecho de conocer y analizar una realidad compleja fomentó la reducción de prejuicios generados hacia los destinatarios por pertenecer a una etnia o grupo de exclusión.

Es una metodología que les enriquece personalmente y favorece la empatía relacional, fomenta el desarrollo de las habilidades sociales y comunicativas, reduce el antagonismo y prejuicio hacia determinados sectores valorando positivamente a los demás, de tal forma que aumenta la sensibilidad y flexibilidad. (Eberly y Roche, 2002, p.4). Además, mejoran su actitud empática al potenciarla desde la intervención en el contexto real. Compartir y trabajar juntos con otras personas de manera sistemática, continua y procesual influye en reforzar los procesos de empatía. (Batlle, 2018).

La mayoría de alumnos universitarios expresaron que el trabajo directo fue complejo porque no todos los destinatarios habían aceptado las dinámicas propuestas relativas a modificar cambios conductuales.

El aprendizaje fue competencial porque adquirieron habilidades en las relaciones interpersonales, ya que los alumnos se adaptaban tanto de manera personal como grupal a las necesidades. Fueron capaces de adquirir herramientas para identificar diferencias y manejar situaciones, resolviendo problemas y gestionando conflictos. Aprendieron a analizar conocimientos teóricos y procesos psicológicos en el contexto donde se desarrollaban conductas individuales y procesos grupales, adaptándose a sus creencias, sus costumbres y cultura. 
Este tipo de metodología favoreció la capacidad de organización y planificación, al tener que organizar los recursos y temporalizar las actividades ajustándose al horario del centro. Trabajaron la capacidad de comunicación oral y aprendieron a trabajar en equipo, no solo en su propio grupo, sino también con diferentes profesionales del centro.

Desarrollaron la competencia de realizar presentaciones audiovisuales al tener que exponer su trabajo mediante recursos audiovisuales. Recopilaron información de libros, de revistas especializadas y de personas para poder elaborar el proyecto.

\section{DISCUSIÓN}

Los cambios que está constantemente experimentando la universidad inciden en que los procesos de innovación metodológica sean fundamentados, sostenibles y prácticos, de manera que el estudiante sea capaz de transformar y analizar críticamente los conocimientos adquiridos. Se fortalecen así las competencias específicas, pero también las transversales como la empatía y la prosocialidad. Esto hace complejo el modo de evaluación de los aprendizajes que trascienden meramente los contenidos específicos y surgen cuestiones concernientes a si un buen proyecto de Aprendizaje-Servicio responde a un logro significativo en los resultados. Por eso, es esencial generar espacios de reflexión durante todo el proceso, pues ésta es una herramienta muy potente para trabajar determinadas actitudes en el alumnado, pero siempre deben estar combinadas con la acción para que se consoliden los comportamientos prosociales (Sandrea y Reyes, 2010, p.397 en Roche, 1995).

Generalmente los estudiantes que participan en experiencias de Aprendizaje-Servicio evalúan más positivamente la asignatura tanto desde una perspectiva académica como personal, dado que perciben de manera más clara el aprendizaje adquirido. Pero muchos de los resultados no pueden ser considerados por una experiencia de Aprendizaje-Servicio, sino que deben ser evaluados a medio o largo plazo como la conducta vinculada a una ciudadanía empática y comprometida (Ruiz-Corbella, 2019, p.18).

La dimensión reflexiva tiene que acompañar todo este proceso para promover, por una parte, un pensamiento crítico, al relacionar el conocimiento teórico-académico y el práctico-experiencial; y por otra, un pensamiento intuitivo, relativo a valores, emociones, habilidades, motivación, vivencias. La dimensión intuitiva y, sobre todo, el factor emocional hace que Aprendizaje-Servicio sea "un aprendizaje de mayor significación personal para el estudiante y esto lo hace más duradero, es decir, menos vulnerable al olvido" (Domingo y Gómez, 2014, p. 50).

El desarrollo personal de participar en experiencias de Aprendizaje-Servicio mejora el compromiso cívico y las habilidades de los estudiantes. Si bien, otras aportaciones aseguran que faltan evidencias empíricas que apoyen las bondades de esta metodología. También hay aspectos negativos porque no todos los alumnos acogen positivamente participar de un proyecto de este tipo. Este aspecto puede responder al hecho de que los alumnos no tengan un nivel cognitivo adecuado para participar en este tipo de acciones y generen así, cierta resistencia. (Deeley, 2016, p. 32-33).

Los estudios de Sandrea, L. y Reyes, L. (2010) demuestran que sí hay resultados positivos respecto al desarrollo de proyectos de Aprendizaje-Servicio y la emisión de conductas prosociales, conclusión que complementa otras investigaciones en las que se afirma que los alumnos son capaces de atender mejor al entorno social y a detectar la necesidad de desarrollar problemas. Sin embargo, otras contribuciones, como la de Billig y otros (2005), concluyeron que no había resultados significativos respecto a la prosocialidad, aunque sus conclusiones no fueron fruto de un programa de intervención. Se propone pues conocer la relación de Aprendizaje-Servicio con la motivación, la experiencia y expectativa que genera el profesor en los estudiantes, así como el tipo de proyecto y la duración. (Sandrea, L. y Reyes, L., 2010).

\section{CONCLUSIONES}

Las experiencias de Aprendizaje-Servicio permiten adquirir competencias curriculares generales de la asignatura y transversales. La participación activa en estos proyectos contribuye a fortalecer el compromiso con la comunidad y la ciudadanía, a aprender habilidades tanto personales como sociales, a desarrollar la sensibilidad 


\section{EMPATÍA Y PROSOCIALIDAD: PROYECTOS DE APRENDIZAJE-SERVICIO EN PSICOLOGÍA SOCIAL}

moral y a definir la identidad personal, desarrollando una mayor capacidad de autonomía e incrementando su autoestima. (Deeley, 2016, p. 29; Puig, 2015, p.24).

Trabajar desde una metodología que genera cierta reciprocidad entre acción-reflexión modula el activismo y academicismo. Cuidar los espacios de reflexión en todas las etapas del proyecto (Rubio y Escofet, 2017, p.85) favorece la adquisición de competencias personales, interpersonales, vocacionales, profesionales, de pensamiento crítico, de ciudadanía y de transformación social. Dicha reflexión queda enmarcada en una pedagogía orientada hacia la autoestima, el altruismo, la solidaridad y la ayuda a los demás. (Puig, 2009, p. 98-100; 119121).

Es necesario elaborar estrategias y rúbricas de seguimiento de todo el proceso. (Ruiz y Novella, 2018, p.1119). Además, conviene recabar información de todos los actores participantes para realizar una evaluación objetiva del impacto del proyecto. (Santos y Lorenzo, 2018, p. 37-40). Los proyectos de Aprendizaje-Servicio debe ser acompañados de un registro sistematizado que genere espacios potentes de reflexión y de elementos concretos que permitan evaluar la calidad de los dinamismos pedagógicos.

\section{REFERENCIAS BIBLIOGRÁFICAS}

Ardila, A. y Correa, G. (2018). (Edts). Observación e investigación para avanzar de lo simple a lo complejo. Aprendizaje-Servicio: dimensiones experienciales de los estudiantes. Universidad de la Salle.

Batlle, R. (2018). "Aprendizaje-Servicio: de la empatía a la fraternidad". Revista Educadores de la Federación de Escuelas Católicas. Número 265.

Deeley, S.J. (2016). El Aprendizaje-Servicio en educación superior. Teoría, práctica y perspectiva crítica. Narcea.

Domingo, A. y Gómez, Mํㅡ. (2014). La práctica reflexiva. Bases, modelos e instrumentos. Madrid: Narcea.

Eberly, D. y Roche, R. (2002). Aprender sirve, servir enseña. Clayss.

Gil, F. y Reyero, D. (Eds.) (2015). Educar en la universidad de hoy. Propuestas para la renovación de la vida universitaria. Madrid: Encuentro. p.50-66.

Martin, X. (2018) Educarse es de valientes. Aprendizaje-Servicio con adolescentes en riego de exclusión social. Octaedro.

Pérez, A. (2007). Educar para humanizar. Narcea.

Puig, J.M. (Coord.) (2009). Aprendizaje-Servicio. Educación y compromiso cívico. Graó.

Puig, J.M., Batlle, R., Bosch, C. y Palos, J. (2006). Aprendizaje-Servicio. Educar para la ciudadanía. Octaedro.

Puig. J.M. (Coord.) (2015). 11 ideas clave. ¿Cómo realizar un proyecto de aprendizaje servicio? Graó.

Rubio, L. y Escofet, A. (coord.). (2017). Aprendizaje-Servicio. Claves para su desarrollo en la universidad. Octaedro.

Ruiz, Y. y Novella, C. (Coord.) (2018). "Claves pedagógicas para la rúbrica de Aprendizaje-Servicio" En El horizonte educativo de la ecología integral. Claves para una cultura del encuentro y la justicia social. Valencia: Universidad Católica de Valencia S. Vicente Mártir. p.7-19.

Ruiz-Corbella, M. y García-Gutiérrez, J. (Eds.) (2019). Aprendizaje-Servicio. Los retos de la evaluación. Narcea.

Sandrea, L. "Proyectos de Aprendizaje-Servicio comunitario: herramienta pedagógica para el fortalecimiento de conductas prosociales" III Congreso y XII Jornadas Científicas "Dres. Eligio Nucette y Lilia Meléndez de Nucette" 1ํal 4 de abril de 2008. Invest Clin 49(Sup. 1): 2008 (p.83-84).

Sandrea, L. y Reyes, L. (2010). "Proyectos de Aprendizaje-Servicio comunitario y su influencia en las conductas prosociales de estudiantes universitarios". Revista de Pedagogía, Vol. 31, № 89. (pp.379-401).

Santos, M.A. y Lorenzo, M (Eds.) (2018) Guía para la institucionalización del Aprendizaje-Servicio universitario. Universidad de Santiago de Compostela. 